\title{
Manejo del nitró geno en la caña de azúcar de la zona centro de Veracruz, México
}

\section{Handling of nitrogen in the sugar zone center of Veracruz, Mexico}

\author{
Landeros-Sánchez, Cesáreo; Moreno-Seceña, Juan Carlos; Castañeda- \\ Chávez, María del Refugio; Lango-Reynoso, Fabiola; Hernández- \\ Pérez, Juan Manuel; Hernández-Lara, Oneyda; Caballero-Hernández, \\ Álvaro José; Editor Académico Dr. Carlos Alberto Zuniga González
}

\author{
Cesáreo Landeros-Sánchez \\ clandero@colpos.mx \\ Colegio de Postgraduados Campus Veracruz, Mexico \\ Juan Carlos Moreno-Seceña \\ secena@colpos.mx \\ Instituto Tecnológico Superior de Martínez de la \\ Torre, Adscripción Xalapa, Mexico \\ María del Refugio Castañeda-Chávez \\ castanedaitboca@yahoo.com.mx \\ Instituto Tecnológico de Boca del Río, Mexico \\ Fabiola Lango-Reynoso \\ fabiolalango@yahoo.com.mx \\ Instituto Tecnológico de Boca del Río, Mexico \\ Juan Manuel Hernández-Pérez \\ juan.hernandez@colpos.mx \\ Colegio de Postgraduados Campus Veracruz, Mexico \\ Oneyda Hernández-Lara \\ Instituto de Geografía Tropical, Cuba \\ Álvaro José Caballero-Hernández \\ Unidad de Investigación Eco intensificación. CICAEA. \\ Departamento de Agroecología. UNAN-León, \\ Nicaragua \\ Editor Académico Dr. Carlos Alberto Zuniga \\ González \\ Universidad Nacional Autónoma de Nicaragua, León, \\ Nicaragua
}

Revista Iberoamericana de Bioeconomía y Cambio Climático

Universidad Nacional Autónoma de Nicaragua, León, Nicaragua ISSN-e: 2410-7980

Periodicidad: Semestral

vol. 2, núm. 1, 2016

czuniga@ct.unanleon.edu.ni

Recepción: 15 Mayo 2015

Aprobación: 13 Noviembre 2015

URL: http://portal.amelica.org/ameli/journal/394/3941750006/
Resumen: El uso excesivo e ineficiente manejo del fertilizante nitrogenado en caña de azúcar (Saccharum officinarum L) causan contaminación de acuíferos. El objetivo de este estudio fue evaluar el rendimiento, rentabilidad y cantidad de nitrógeno lixiviado en caña de azúcar, bajo la aplicación fraccionada del nitrógeno total que se aplica a la caña de azúcar. El estudio se realizó en el Módulo de Riego I-1 La Antigua, Veracruz, México. Se evaluaron nueve tratamientos que resultaron de los factores: dosis 250,200 y $150 \mathrm{~kg} \mathrm{ha}^{-1}$ de $\mathrm{N}$ y fraccionamientos 2, 3 y 4. Las variables de respuesta fueron: rendimiento, relación Beneficio/Costo y lixiviación de N. Se encontró una actitud ligeramente positiva (3.3) en escala Likert hacia la reducción de la dosis y aumento en el fraccionamiento del $\mathrm{N}$ total que se aplica al cultivo. Para el rendimiento, solo el fraccionamiento mostró diferencias significativas; al fraccionar el nitrógeno en tres y cuatro aplicaciones se obtuvieron rendimientos superiores a $125 \mathrm{t} \mathrm{ha}^{-1}$. Las menores pérdidas por lixiviación se registraron con una dosis de $150 \mathrm{~kg} \mathrm{ha}^{-1}$ de $\mathrm{N}$ fraccionada en tres y cuatro aplicaciones, esto es, 16.80 y $15.40 \mathrm{~kg} \mathrm{ha}^{-1}$ de $\mathrm{N}$ respectivamente. Se concluyó que el empleo de dosis de $150 \mathrm{~kg} \mathrm{ha}^{-1}$ y con tres fraccionamientos del $\mathrm{N}$ total aplicado, representa una alternativa más sustentable para la producción de caña de azúcar. Por lo tanto, este manejo puede resultar en una mayor absorción de $\mathrm{N}$ por el cultivo y menos $\mathrm{N}$ se lixiviará hacia el acuífero.

Palabras clave: Nitrógeno, contaminación de acuífero, agroecosistema caña de azúcar.

Abstract: The excessive use of nitrogen in sugarcane (Saccharum officinarum L) is a source of contamination of aquifers. The objective of this study was to evaluate the yield, profitability and leaching losses of nitrogen $(\mathrm{N})$ in sugarcane plantations when the total nitrogen applied is fractionized in several applications. The study was carried out at the "Módulo de Riego I-1 La Antigua”, Veracruz, Mexico. Nine treatments were evaluated, that resulted from the factors: amount of nitrogen applied (250, 200 y $150 \mathrm{~kg} \mathrm{ha}^{-1}$ ) and fractioning of nitrogen applied (2, 3 and 4 applications). The response variables were yield, costbenefit ratio and nitrogen leaching. It was found that only the 
DOI: https://doi.org/10.5377/ribcc.v2i1.5677

Autor de correspondencia: clandero@colpos.mx nitrogen fractioning showed significant differences for 3 and 4 applications, which resulted in higher yield than $125 \mathrm{t} \mathrm{ha}^{-1}$. The best cost-benefit ratio was found with a dose of $150 \mathrm{~kg} \mathrm{ha}^{-1}$ of $\mathrm{N}$ fractionized in 3 applications. The lowest nitrogen leaching losses were recorded for $150 \mathrm{~kg} \mathrm{ha}^{-1}$ of $\mathrm{N}$ fractionized in 3 and 4 applications. Therefore, this nitrogen fertilizer management may result in a higher nitrogen absorption by the crop and less nitrogen will be leached in to the aquifer.

Keywords: Nitrogen, aquifer contamination, sugar cane agroecosystem.

\section{INTRODUCCIÓN}

Uno de los principales insumos que requiere la caña de azúcar para su buen desarrollo es el fertilizante nitrogenado. Por lo que este nutrimento debe aplicarse en las dosis requeridas por este cultivo, a fin de obtener rendimientos por unidad de superficie que resulten en mejores beneficios financieros para los productores (Segura et al., 2000; Smith et al., 2000, Weterings y Russell, 2004). Además, la cantidad total de nitrógeno (N) que demanda el cultivo debe distribuirse de acuerdo a las necesidades que requiere la planta en sus fases de crecimiento y desarrollo (Gowda et al., 1988; Toledo et al., 2002). El nitrógeno es un elemento muy móvil y está expuesto a pérdidas por lixiviación, volatilización y desnitrificación. Por lo que es importante incorporarlo al suelo fraccionado, para que esté disponible en las diversas etapas fenológicas del cultivo (Hurtado et al., 2007). Además, el uso ineficiente y excesivo del nitrógeno incrementa el costo de producción y puede ser una fuente de contaminación de nitratos en mantos freáticos (Yepis et al., 1999). La principal preocupación de esta contaminación estriba en los impactos negativos originados a la salud humana que resulta del consumo de esta agua, ya que se han relacionado casos de cáncer al respecto (Pretty \& Conway, 1998 y FAO, 1997).

El manejo de agua de riego ineficiente y fertilizante nitrogenado que se practica a nivel parcelario contribuye a que el problema de lixiviación de $\mathrm{N}$ hacia el acuífero y corrientes superficiales sea mayor. Así, Castro-Luna et al. (2006) indicaron la necesidad de desarrollar y establecer prácticas alternativas de fertilización sustentables que sean económicas y más eficientes, ya que existe evidencia de que el cultivo de la caña de azúcar aprovecha solamente el $57 \%$ del N que se aplica con el manejo tradicional antes referido (Landeros-Sánchez et al., 2007).

El Ejido Salmoral, pertenece a la zona del Módulo de Riego I-1 La Antigua, Veracruz, México, donde la fertilización nitrogenada se ha incrementado paulatinamente hasta situarse en los $254 \mathrm{~kg} \mathrm{ha}^{-1}$, en promedio. Lo anterior, sin considerar la variedad de caña, el tipo de suelo y disponibilidad de agua. Comúnmente se utilizan fuentes como Urea (46-0-0) y mezclas como 20-10-10; es usual que el $\mathrm{N}$ total que se suministra al cultivo se haga solamente en una aplicación o fraccionado en dos aplicaciones, y extemporáneamente, debido al retraso en la adquisición del fertilizante cuando éste se obtiene vía crédito (Moreno-Seceña et al., 2007). Países como Brasil y Cuba, que llegaron aplicar hasta $350 \mathrm{~kg} \mathrm{ha}^{-1} \mathrm{de} \mathrm{N}$ a la caña de azucar, redujeron desde hace dos decadas sus dosis de fertilización de $\mathrm{N}$; actualmente aplican entre 50 y $100 \mathrm{~kg} \mathrm{ha}^{-1}$ (Dematté et al., 2005). Como resultado de lo anterior, sus rendimientos de campo se han mantenido y son similares a los de

\section{NotAS DE AUTOR}


zafras anteriores, y a aquellos registrados en otros paises como México, donde actualmente se aplican dosis de $\mathrm{N}$ superiores a los $180 \mathrm{~kg} \mathrm{ha}^{-1}$ en promedio.

No solamente el nitrógeno es absorbido en cantidades limitadas por las plantas, como se mencionó anteriormente, los otros fertilizantes presentan problemas similares. Se sabe que las plantas solo absorben el $20 \%$ del fósforo aplicado a los cultivos y el resto se fija, en formas insolubles, en el suelo. Por tanto, el objetivo de este estudio fue evaluar el rendimiento, rentabilidad y cantidad de nitrógeno lixiviado en caña de azúcar, bajo la aplicación fraccionada del nitrógeno total que se aplica a la caña de azúcar.

\section{Materiales y MÉTODOS}

El experimento se desarrolló de enero a diciembre de 2009, la parcela experimental se ubicó en el ejido Salmoral, municipio de La Antigua, Veracruz, México. Los suelos son de tipo aluvial, propios de la región, con textura migajón arcilloso y manto freático de 75 a $95 \mathrm{~m}$ de profundidad. La variedad de caña de azúcar cultivada fue la CP 72-2086, en su ciclo de producción soca. Se evaluaron nueve tratamientos (Tabla I) combinación de los factores siguientes: dosis $\left(250,200\right.$ y $\left.150 \mathrm{~kg} \mathrm{ha}^{-1} \mathrm{de} \mathrm{N}\right)$ y fraccionamiento (2, 3 y 4$)$. La primera aplicación del fertilizante fue a los cinco días del rebrote de la plántula y las siguientes a los 30, 60 y 90 días después de la primera aplicación. El sistema nutricional de la planta de todos los tratamientos se balanceó adicionando fósforo $\left(\mathrm{P}_{2} \mathrm{O}_{5}\right)$ y potasio $\left(\mathrm{K}_{2} \mathrm{O}\right)$, a razón de 20 y $60 \mathrm{~kg} \mathrm{ha}^{-1}$. Se aplicaron seis riegos en las unidades experimentales y en los nueve lisímetros instalados, durante el ciclo del cultivo, con una lámina de $15 \mathrm{~cm}$. Se utilizó un diseño experimental en campo de bloques completamente al azar. Las variables de respuesta fueron: rendimiento $\left(\mathrm{t} \mathrm{ha}^{-1}\right)$ al momento de cosecha del cultivo, Relación beneficio/costo (R: B/ C) como indicador de rentabilidad. Como beneficio (B) se consideró el precio del rendimiento obtenido $\left(\mathrm{t} \mathrm{ha}^{-1}\right)$, y para el costo $(\mathrm{C})$ se consideraron los costos totales de la producción. La lixiviación del nitrógeno se midió instalando nueve lisímetros de balance en los que se ensayaron y replicaron las condiciones de los tratamientos en campo.

TABLA I.

Tratamientos evaluados para estudiar el efecto de dosis y fraccionamiento del nitrógeno.

\begin{tabular}{|c|c|c|c|}
\hline $\mathrm{N}^{\circ}$ & Clave & $\begin{array}{c}\text { Dosis } \\
\left(\mathrm{kg} \mathrm{N} \mathrm{ha}^{-1}\right)\end{array}$ & Fraccionamiento aplicado \\
\hline 1 & ${ }^{\mathrm{T}} \mathrm{D} 1 \mathrm{~F} 1$ & 250 & Dos aplicaciones (125 y 125 ). \\
\hline 2 & $\mathrm{D} 1 \mathrm{~F} 2$ & 250 & Tres aplicaciones $(80,90$ y 80$)$. \\
\hline 3 & D1F3 & 250 & Cuatro aplicaciones $(60,70,60$ y 60$)$ \\
\hline 4 & $\mathrm{D} 2 \mathrm{~F} 1$ & 200 & Dos aplicaciones (100 y 100$)$. \\
\hline 5 & $\mathrm{D} 2 \mathrm{~F} 2$ & 200 & Tres aplicaciones $(70,70$ y 60$)$. \\
\hline 6 & $\mathrm{D} 2 \mathrm{~F} 3$ & 200 & Cuatro aplicaciones $(50,50,50$ y 50$)$ \\
\hline 7 & $\mathrm{D} 3 \mathrm{~F} 1$ & 150 & Dos aplicaciones (75 y 75$)$ \\
\hline 8 & $\mathrm{D} 3 \mathrm{~F} 2$ & 150 & Tres aplicaciones $(50,50$ y 50$)$. \\
\hline 9 & D3F3 & 150 & Cuatro aplicaciones $(40,40,40$ y 30$)$ \\
\hline
\end{tabular}

T Testigo, que corresponde a la dosis y aplicación recomendada por los Ingenios.

elaboración propia 


\section{RESULTADOS Y DISCUSIÓN}

Rendimiento en campo. Los rendimientos obtenidos para cada uno de los tratamientos evaluados, fueron superiores a los de la media de la región que es de $100.1 \mathrm{tha}^{-1}$ (Figura 1).

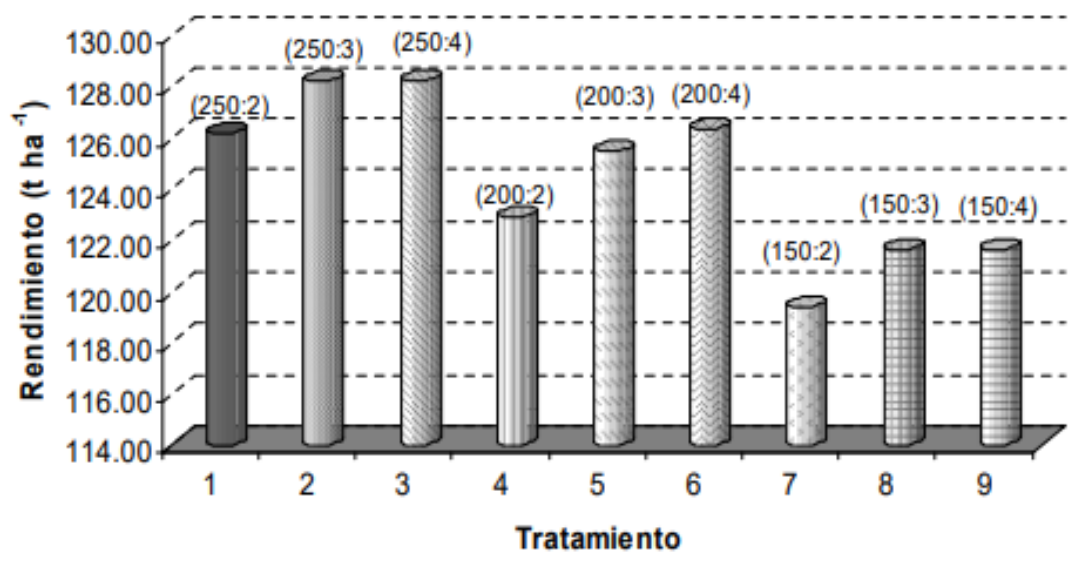

FIGURA 1.

Rendimientos obtenidos en el cultivo de la caña de azúcar. Entre paréntesis la dosis de nitrógeno y el fraccionamiento aplicado.

elaboracion propia

El análisis de varianza mostró que el factor dosis no causó diferencias significativas en los rendimientos obtenidos. Lo que significa que el productor al utilizar una dosis menor de fertilizante no tendrá impactos negativos en el rendimiento del cultivo. Sin embargo, el factor fraccionamiento del nitrógeno si causó diferencias significativas en los rendimientos obtenidos. La Tabla II muestra la comparación de medias entre factores estudiados; además indica que al fraccionar la cantidad total de nitrógeno aplicado al cultivo de caña de azúcar, en tres y cuatro aplicaciones, se obtienen rendimientos estadísticamente similares y superiores a $125 \mathrm{tha}^{-1}$.

TABLA II.

Comparación de rendimientos medios en caña de azúcar, en relación al factor dosis y fraccionamiento de $\mathrm{N}$.

\begin{tabular}{ccccc}
\hline & \multicolumn{4}{c}{ Número de Fraccionamientos } \\
\cline { 2 - 5 } Dosis de N $\left(\mathbf{k g ~ h} \mathbf{~ h}^{-1}\right)$ & $\mathbf{2}$ & $\mathbf{3}$ & $\mathbf{4}$ & Promedio \\
\hline 250 & 126.17 & 128.20 & 128.20 & $127.52 \mathrm{a}$ \\
200 & 122.90 & 125.50 & 126.33 & $124.90 \mathrm{a}$ \\
150 & 119.40 & 121.63 & 121.63 & $120.88 \mathrm{a}$ \\
Promedio & $\mathbf{1 2 2 . 8 2} \mathbf{b}$ & $\mathbf{1 2 5 . 1 0} \mathbf{a}$ & $\mathbf{1 2 5 . 3 8} \mathbf{a}$ & \\
\hline
\end{tabular}

Letras diferentes entre columnas indican diferencias estadísticas significativas (Tukey, $\mathrm{P} \leq 0.05$ ).

elaboracion propia

Los resultados obtenidos mostraron que al aplicar dosis de fertilización superiores a los $150 \mathrm{~kg} \mathrm{ha}^{-1}$ de nitrógeno, no se garantiza mayores rendimientos. Sin embargo, el fraccionamiento generó mayores rendimientos en el cultivo. De acuerdo con Pacheco (1992) y Weier et al. (1996) el fraccionamiento del fertilizante nitrogenado aumenta la eficiencia de absorción del cultivo.

Relacion Beneficio-Costo: De acuerdo a las medias obtenidas para los tratamietos evaluados, el tratamiento que mostro la mayor relacion beneficio/costo, fue el tratamiento ocho (150:3) a razón de 1.80. La menor relación beneficio-costo se obtuvo en el tratamiento uno (250:2), la cual fue de 1.67. De acuerdo 
al análisis de varianza, el factor dosis provocó diferencias estadísticas en la relación beneficio/costo. Lo anterior se debió a que el importante ahorro alcanzado en las dosis de nitrógeno, se tradujo en un ahorro económico considerable, lo cual permitió elevar la relación beneficio/costo de los tratamientos con menor dosis de nitrógeno (Tabla III). El factor fraccionamiento no produjo diferencias estadísticas significativas en el beneficio/costo.

TABLA III.

Comparación de medias de la relación Beneficio/Costo, obtenidas en caña de azúcar, en relación con los factor dosis y fraccionamiento de $\mathrm{N}$.

\begin{tabular}{ccccc}
\hline & \multicolumn{5}{c}{ Número de Fraccionamientos } \\
\cline { 2 - 5 } Dosis de $\mathbf{N}\left(\mathbf{k g ~ h a}^{-1}\right)$ & $\mathbf{2}$ & $\mathbf{3}$ & $\mathbf{4}$ & Promedio \\
\hline 250 & 1.67 & 1.70 & 1.71 & $\mathbf{1 . 6 9} \mathbf{~ b}$ \\
200 & 1.73 & 1.76 & 1.77 & $\mathbf{1 . 7 5} \mathbf{~ a}$ \\
150 & 1.77 & 1.80 & 1.79 & $\mathbf{1 . 7 9} \mathbf{a}$ \\
Promedio & $1.72 \mathrm{a}$ & $1.75 \mathrm{a}$ & $1.76 \mathrm{a}$ & \\
\hline
\end{tabular}

Letras diferentes entre hileras indican diferencias estadísticas significativas (Tukey, $\mathrm{P} \leq 0.05$ ).

Con base en los resultados obtenidos para la variable relación beneficio/costo, se observa que al utilizar una dosis de $150 \mathrm{~kg} \mathrm{ha}^{-1} \mathrm{de} \mathrm{N}$, fraccionado en tres aplicaciones, se genera una mayor rentabilidad en la producción de caña de azúcar. Al respecto, Wilcox (1991) mencionó que la reducción de la dosis de N, el fraccionamiento y el uso de residuos orgánicos como vinaza y la biomasa producida por el mismo cultivo; son alternativas viables para la reducción de los costos de la fertilización. Sin embargo, Hernández et al. (2008), después de realizar un análisis económico del empleo de dosis reducidas de fertilización nitrogenada en combinación con vinaza y cachaza, llegaron a la conclusión de que la fertilización química es la opción más rentable; pero esta omite los efectos benéficos residuales que ejercen la composta y vinaza sobre el suelo. Por lo anterior, es necesario considerar no solamente la reducción de las dosis y fraccionamiento del fertilizante nitrogenado, sino también la incorporación de residuos de cosecha o residuos orgánicos como fuentes de nitrógeno para el desarrollo de la caña de azúcar.

Lixiviación de nitrógeno: Se encontró que al aplicar una dosis menor de nitrógeno, las pérdidas de este nutrimento por lixiviación son menores. Lo anterior debido a que cuando se aplica una dosis de $250 \mathrm{~kg}$ $\mathrm{ha}^{-1}$ de $\mathrm{N}$, fraccionada en dos, tres y cuatro aplicaciones, se pierden de manera acumulada de 30.62 hasta $40.86 \mathrm{~kg}$ de nitrógeno por hectárea. Cuando se aplicó una dosis de $200 \mathrm{~kg} \mathrm{ha}^{-1} \mathrm{de} \mathrm{N}$, fraccionada en dos, tres y cuatro aplicaciones, se registró una pérdida acumulada que osciló de 21.82 a $30.16 \mathrm{~kg}$ de nitrógeno por hectárea, lo que representó una pérdida de hasta el $15.0 \%$. Lo anterior coincide con lo reportado por Bergström y Johansson (1991), quienes reportaron pérdidas por lixiviación de $\mathrm{N}$ del orden de este porcentaje para la misma dosis. Al aplicar una dosis de $150 \mathrm{~kg} \mathrm{ha}^{-1}$ de nitrógeno, fraccionándolo en dos, tres y cuatro aplicaciones, se observa que las pérdidas de $\mathrm{N}$ oscilan entre $15.40 \mathrm{a} 18.18 \mathrm{~kg} \mathrm{ha}^{-1}$, lo cual representa una perdida de hasta el $12.1 \%$ (Figura 2). La menor pérdida de $\mathrm{N}$ se presentó en el tratamiento nueve. Esto coincidió con lo reportado por Chavez (1999), quien señaló que el fraccionamiento de la fertilizacion nitrogenada es una práctica que reduce la perdida de nitrógeno por lixiviación. 


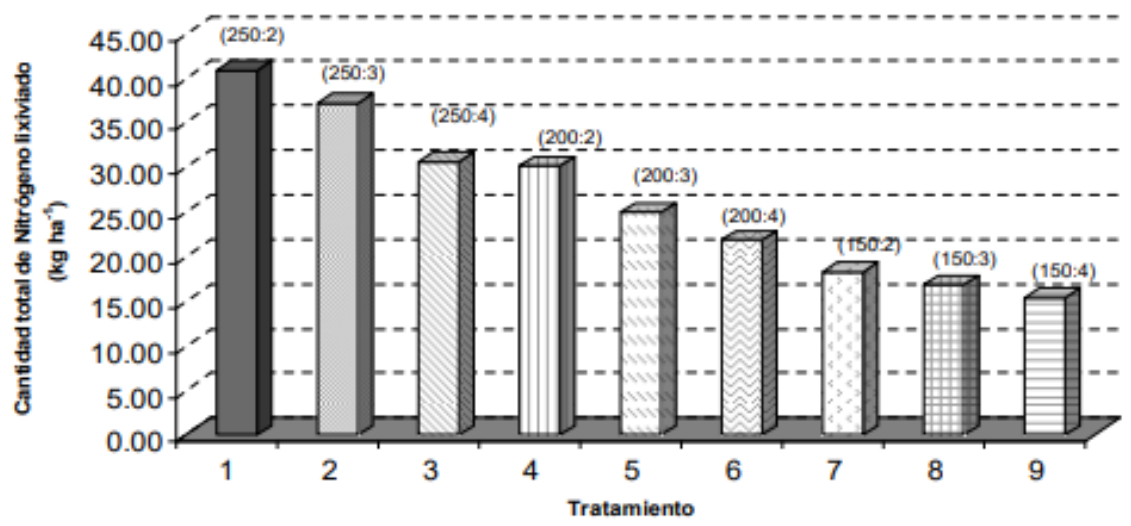

FIGURA 2.

Lixiviación acumulada total de $\mathrm{N}$ registrada en lisímetros. En paréntesis: dosis de $\mathrm{N}$ (kg ha-1) y fraccionamiento aplicado. elaboracion propia

La Figura 3 muestra el comportamiento temporal de la lixiviación de $\mathrm{N}$ registrada.

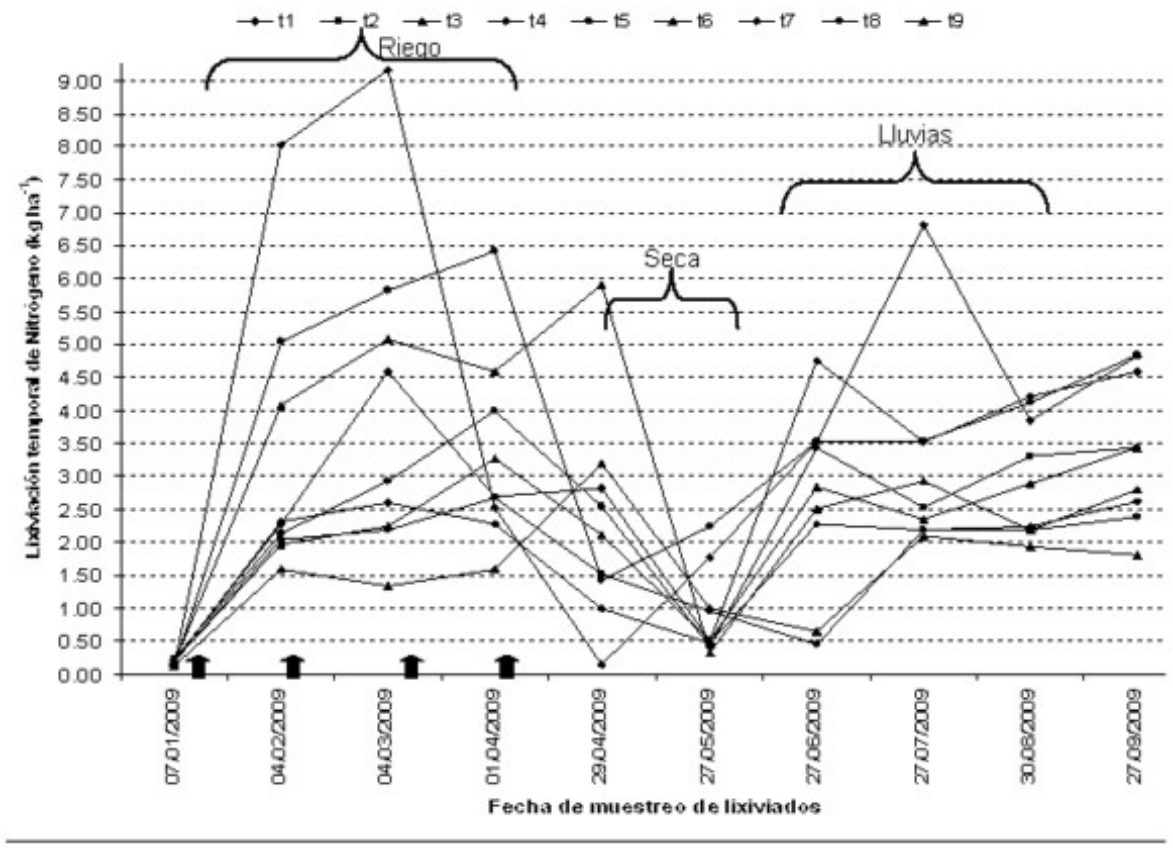

FIGURA 3.

Comportamiento temporal de lixiviación de N. Las

flechas indican el momento de aplicación de fertilizante. elaboracion propia

En relación con los resultados de lixiviación de N que se muestran en la Figura 3, se observa que el manejo del agua juega un papel muy importante en este proceso, ya que se registraron las mayores lixiviaciones en la época de riego, seguido por aquellas ocasionadas por las lluvias y las menores en épocas de seca. Sin embargo, en todas las épocas, las mayores dosis fueron las que registraron las mayores lixiviaciones de $\mathrm{N}$.

En la Tabla IV se integran los resultados de las variables evaluadas en este estudio, de los cuales se genera una recomendación de fertilización y manejo regional. Asimismo, se observa que el tratamiento más adecuado para recomendar a los productores cañeros del Módulo I-1 La Antigua, Veracruz, es el tratamiento ocho, 
con el cual se utiliza una dosis de $150 \mathrm{~kg} \mathrm{ha}^{-1}$ de $\mathrm{N}$, fraccionado en tres aplicaciones. Con esto se obtendrían los mayores beneficios económicos y ambientales, traducidos en menor impacto por contaminación de nitrógeno al acuífero. Por tanto, para mejorar la fertilidad natural del suelo y los rendimientos por unidad de superficie, se recomienda la incorporación de residuos de cosecha del cultivo y un manejo eficiente del agua de riego.

\begin{tabular}{cccccc}
\hline & \multicolumn{2}{c}{ Variables: } & Productiva & Económica & Ambiental \\
\hline To. & $\begin{array}{c}\text { Dosis de N } \\
\left(\mathrm{kg} \mathrm{ha}^{-1}\right)\end{array}$ & Fraccionamiento & $\begin{array}{c}\text { Rendimiento } \\
\left(\mathrm{t} \mathrm{ha}^{-1}\right)\end{array}$ & $\begin{array}{c}\text { Rixiviación de N } \\
\left(\mathrm{kg} \mathrm{ha}^{-1}\right)\end{array}$ \\
\hline 1 & 250 & 2 & 126.1 & 1.67 & 40.9 \\
2 & 250 & 3 & 128.2 & 1.70 & 37.2 \\
3 & 250 & 4 & 128.2 & 1.71 & 30.6 \\
4 & 200 & 2 & 122.9 & 1.73 & 30.1 \\
5 & 200 & 3 & 125.5 & 1.76 & 24.9 \\
6 & 200 & 4 & 126.3 & 1.77 & 21.8 \\
7 & 150 & 2 & 119.4 & 1.77 & 18.1 \\
$* 8$ & 150 & 3 & 121.6 & 1.80 & 16.8 \\
9 & 150 & 4 & 121.6 & 1.79 & 15.4 \\
\hline * Tratamiento adecuado al objetivo del estudio. & & &
\end{tabular}

FIGURA IV.

Variables productivas, económicas y ambientales estudiadas en caña de azúcar. elaboracion propia

\section{Cómo mejorar el manejo del fertilizante y reducir la contaminación del acuífero y corrientes naturales}

Una de las alternativas prácticas y realistas gira en torno a la capacitación de técnicos y productores en relación con el uso de cantidades de fertilizante nitrogenado que la planta demanda para su desarrollo, esto es, reducir el uso de cantidades excesivas de dicho fertilizante; asimismo, capacitarlos sobre la práctica de un manejo eficiente del agua de riego a nivel parcelario. Es necesario darles a conocer las bondades de los sistemas de riego presurizados modernos y orientarlos en cómo acceder a los apoyos gubernamentales que están enfocados a contribuir al desarrollo y modernización de la infraestructura hidroagrícola. También es importante informarlos sobre los impactos negativos que tienen los fertilizantes nitrogenados que se aplican en exceso, los cuales eventualmente se lixivian hacia el manto freático y acuífero profundo, y se transportan hacia cuerpos de agua receptores. Las aguas contaminadas con nitrógeno afectan a la salud humana, fauna y flora silvestre, ecosistemas acuáticos y al medio ambiente en general. Por otro lado es importante capacitarlos sobre los beneficios de usar fertilizantes orgánicos en lugar de formas minerales. Asimismo, introducir la aplicación de fertilizantes nitrogenados de liberación gradual para extender el tiempo de su disponibilidad en la zona radical de la planta, lo cual ocurre de manera similar cuando se aplica composta orgánica. Es importante reintroducir la no quema de la caña de azúcar antes y después de la cosecha, a fin de aprovechar el nitrógeno contenido en hojas y cogollos que se queman. Es necesario formarlos también en temas sobre el cuidado del medio ambiente y los impactos que la quema de caña de azúcar tiene sobre el fenómeno del cambio climático.

\section{CONCLUSIONES}

El manejo del fertilizante nitrogenado mediante aplicaciones fraccionadas a la caña de azúcar, induce una mayor absorción de este nutrimento por el cultivo reflejandose en un aumento en los rendimietos de campo. Además, la aplicación de dosis del fertilizante nitrogenado menores resultó en ahorros económicos y en menores pérdidas por lixiviación hacia el manto freático. Asimismo, mediante el enfoque de este estudio, se logró identificar la dosis y el manejo productivo, económico y ambiental del fertilizante nitrogenado 
que resulta en mejores rendimientos y beneficios financieros al productor cañero, y al ambiente en el agroecosistema con caña de azúcar en el Módulo de Riego I-1 La Antigua, Veracruz, México. Esto es, la aplicación de una dosis de $150 \mathrm{~kg} \mathrm{ha}^{-1}$ de nitrógeno, fraccionado en tres aplicaciones, puede mejorar los rendimientos y ayudar a mitigar el efecto de contaminación de mantos freáticos provocado por nitrógeno, lo cual impacta negativamente la salud humana y al medio ambiente en general.

La lixiviación de $\mathrm{N}$ se ve favorecida con grandes cantidades de agua aportada al suelo, por lo que el manejo eficiente del agua juega un papel importante para mitigar este problema. Además, se pueden adoptar, a nivel de campo, otras medidas sustentables para contrarrestar el problema bajo estudio, particularmente si se establece la cultura de la no quema de la caña de azúcar y la utilización de residuos de la cosecha y otros materiales orgánicos, como fuentes de $\mathrm{N}$.

\section{Agradecimientos}

Al Colegio de Postgraduados, Fideicomiso No. 167304 y Líneas de Investigación en Agroecosistemas Sustentables (LPI2) e Impacto y Mitigación del Cambio Climático (LPI8). Al Consejo Nacional de Ciencia y Tecnología por el financiamiento de este proyecto, clave PM-908-45.

\section{REFERENCIAS}

Bergström, L; Johansson, R. (1991). Leaching of nitrate from Monolith Lysimeters of Different Types of Agricultural Soils. Journal of Enviromental Quality. 20: 801-807.

Castro-Luna, I., Gavi-Reyes, F., Peña-Cabriales, J. J., Núñez-Escobar, R., \& Etchevers-Barra, J. D. (2006). Eficiencia de recuperación de N y K de tres fertilizantes de lenta liberación. Terra Latinoamericana, 24(2), 277-282.

Chávez, M. (1999). Nutrición y fertilización de la caña de azúcar en Costa Rica. Conferencia 78. Dirección de Investigación y Extensión de la Caña de Azúcar (LAICA-DIECA), San José, 43

Demattê,J. A. M., Silva, M. L. D. S., Rocha, G. C., Carvalho, L. A. D., Formaggio, A. R., \& Firme, L. P. (2005). Variações espectrais em solos submetidos à aplicação de torta de filtro. Revista Brasileira de Ciência do Solo, 29(3), 317-326.

FAO. (1997). http://www.fao.org/docrep/W2598S/w2598s00.htm\#Contents

Hurtado, M. G., Díaz, M. I. H., Martell, D. D., Briones, J. R., Acosta, C. R., Cuesta, E., \& Sardiña, C. (2007). Síntesis y comportamiento de un material polimérico aplicado como recubrimiento en un fertilizante de liberación controlada. Revista Iberoamericana de Polimeros, 8(4), 275-286.

Gowda, J. V., Jayanthi, R., \& Raju, B. (1988). Studies on the effects of nitrogen and phosphorus on flowering in gladiolus. Current Research University of Agricultural Science, 17(16), 80-81.

Hernández Melchor, G. I., Salgado García, S., Palma López, D. J., Lagunes Espinoza, L. D. C., Castelán Estrada, M., \& Ruiz Rosado, O. (2008). Vinaza y composta de cachaza como fuente de nutrientes en caña de azúcar en un gleysol mólico de Chiapas, México. Interciencia, 33(11), 855-860.

Landeros-Sánchez, C., Castañeda, C. M., Lango, R. F., Moreno-Seceña, J. C., \& Palomarez, G. M. (2007). Contaminación del agua por nitrógeno causada por la agricultura en zonas cañeras de Veracruz, México. In Memoria del IX Simposio Internacional y IV Congreso Nacional de Agricultura Sostenible Veracruz. Boca del Río, Veracruz, México. Noviembre (pp. 19-26).

Moreno-Seceña J; Landeros S. C; Pérez V, A; López-Collado J; Palacios Vélez O; Ma Del RefugioCastañeda. (2007). Uso y manejo de fertilizantes en caña de azúcar en la zona de abasto de losingenios La Gloria y El Modelo. Memoria del IX Simposio Internacional y IV CongresoNacional de Agricultura Sostenible Veracruz. Boca del Rio, Veracruz. Noviembre. 372p.

Pacheco, A. J. (1992). Nitratos en agua subterránea. Ciencia y desarrollo 17 (102), 98-104.

Pretty, J. N., \& Conway, G. R. (1988). blue-baby syndrome and nitrogen fertilisers: a high risk in the tropics. Gatekeeper series-SA. 
Segura, M. L., Cortés, E. M., \& Sánchez, E. M. C. (2000). Fertilización y riego bajo invernadero en producción integrada. Horticultura: Revista de industria, distribución y socioeconomia horticola: frutas, hortalizas, flores, plantas, árboles ornamentales y viveros, (146), 16-24.

Smith, C. J., Dunin, F. X., Poss, R., \& Angus, J. F. (2000). Nitrogen budget of wheat growing on a Riverine clay soil. Australian Journal of Agricultural Research, 51(7), 867-876.

Toledo, Y., Hernández, A., Alvarez, M., Martín, G. M., \& Márquez, R. (2002). Determinación del efecto antagónico de un biopreparado a partir de una cepa de Burkholderia cepacia ante Fusarium sp en el cultivo del gladiolo (Gladiolus sp). Cultivos tropicales, 23(4), 11-15.

Weterings, K., \& Russell, S. D. (2004). Experimental analysis of the fertilization process. The Plant Cell, 16(suppl_1), S107-S118.

Weier, K. L., McEwan, C. W., Vallis, I., Catchpoole, V. R., \& Myers, R. J. (1996). Potential for biological denitrification of fertilizer nitrogen in sugarcane soils. Australian Journal of Agricultural Research, 47(1), 67-79.

Wilcox, T. (1991). Fertilizer selection strategy may reduce production costs. Bureau Sugarcane Experimental Station. Australia. Bulletin, (35), 7-8.

Yepis Vargas, O; Fundora Herrera, O; Pereira Marín, C; Crespo Borges, T. (1999). La contaminaciónambiental por el uso excesivo de fertilizantes nitrogenados en el cultivo de tomate. Scientia Gerundensis. 24: 5-12. 